\title{
Otogenic tetanus: A challenge for anesthetic management
}

\author{
Jyoti V Kulkarni, Anil Shriniwas Joshi, Rashmi Bengali, \\ Suhas Jewalikar
}

\begin{abstract}
Introduction: Tetanus is a disease caused by endotoxins, tetanolysin and tetanospasmin released from Clostridium tetani which affects motor inhibitory neurons. The challenge for anesthesiologist lies in control of autonomic dysfunction and muscle spasticity. Case Report: In this article we have discussed anesthetic management of a 5-year-old boy with otogenic tetanus posted for removal of foreign body in left ear. He was admitted in infectious ward with trismus, fever, muscle spasm and difficulty in swallowing for last four days. He received intramuscular tetanus toxoid, intramuscular immunoglobulin 5000 IU as bolus followed by $2500 \mathrm{IU} /$ day. Intravenous diazepam $6 \mathrm{mg}$ at every three hours and intravenous cefixime was given. On second day of admission he was posted for removal of foreign body in left ear. Intravenous diazepam and fentanyl were given as premedication and sevoflurane, nitrous oxide and oxygen was used for general anesthesia. During surgery and in postoperative period he developed hypertension and tachycardia, was treated with intravenous labetolol. Conclusion:
\end{abstract}

Jyoti V Kulkarni ${ }^{1}$, Anil Shriniwas Joshi², Rashmi Bengali, Suhas Jewalikar ${ }^{3}$

Affiliations: ${ }^{1}$ Assistant Professor, MD, (Aneasthesiology), Department of Anaesthesia, Govt. Medical College and Hospital, Aurangabad, Maharashtra, India; ${ }^{2}$ Assistant Professor, MD, Medicine, \& Dept. of Medicine, Govt. Medical College and Hospital, Aurangabad, Maharashtra, India; ${ }^{3}$ Associate Professor, MD, Anaesthesia, Dept. of Anaesthesia, Govt. Medical College and Hospital, Aurangabad, Maharashtra, India.

Corresponding Author: Dr. Jyoti V Kulkarni, 7, Bharat Nagar, Near Jyoti Mandir, Shahanoorwadi, Aurangabad, 431005, Phone: 919822769648; E-mail: jyotianil.joshi71@gmail.com

Received: 30 July 2012

Accepted: 16 November 2012

Published: 01 April 2013
In case of tetanus, volatile anesthetic agents can be safely used for surgical procedures like cleaning and debridement of wound, removal of foreign body or incision and drainage of abscess. However, use of nondepolarizing muscle relaxant is advisable. As we used sevoflurene in single case, further study is required for confirmation of its efficacy and safety. Autonomic hyperactivity can be managed with beta-blocker, intravenous labetolol is the drug of choice.

Keywords: Otogenic tetanus, Sevoflurane, Labetolol, Foreign body

$$
* * * * * * * * *
$$

Kulkarni JV, Joshi AS, Bengali R, Jewalikar S. Otogenic tetanus: A challenge for anesthetic management. International Journal of Case Reports and Images 2013;4(4):232-235.

$$
* * * * * * * * *
$$

doi:10.5348/ijcri-2013-04-301-CR-10

\section{INTRODUCTION}

Tetanus is an infectious disease caused by anaerobic spore forming bacteria Clostridium tetani $[1,2]$. Incidence of tetanus is higher in males than in females [3]. Clinical presentation is due to toxins produced by the organisms, tetanospasmin and tetanolysin. The portals of entry of the bacteria into the body is usually contaminated wound. When a suppurating ear is the only known portal of entry, it is termed otogenic tetanus [4]. Otogenic tetanus is more common in children less than 6 years [4]. Mortality is lower in otogenic tetanus than in other groups [3]. In tetanus, mortality is due to respiratory failure and cardiovascular collapse, associated with autonomic instability [5]. 
Tetanospasmin inhibits release of neurotransmitter from presynaptic inhibitory neurons resulting in reflex irritability and autonomic hyperactivity [6]. Respiratory failure is secondary to muscle rigidity, reflex muscle spasm and high levels of circulating catecholamine or toxic myocarditis [6]. Parasympathetic over activity leading to bradycardia and sinus arrest is also known to occur in severe cases [6].

For better outcome, these patients should be treated in intensive care unit. Neutralization of toxin load by antitetnus globulin, airway maintainance, eradication of source of toxin, control of muscle spasm and autonomic instability are the goals in treating these patients. No specific protocols are given regarding anesthetic management of patients of tetanus. Deeper plane of anesthesia with paralysed patient is preferred to prevent muscle spasm and hypertensive crisis [2]. Nondepolarizing muscle relaxants can be safely used but higher doses may be needed. Depolarizing muscle relaxants should be used with caution as it may trigger hyperkalemic arrest. Volatile anesthetic agents can be safely used.

Tracheostomy or supraglottic airway is preferred over endotracheal intubation. Autonomic hyperactivity can be managed with beta-blockers or by epidural or intrathecal anesthesia [4]. Our patient was posted for removal of foreign body in ear. We used intravenous diazepam and fentanyl as premedication and anesthesia was given with inhalational agents' sevoflurane, nitrous oxide and oxygen. Intravenous labetolol was used to combat sympathetic over activity.

\section{CASE REPORT}

A 5-year-old boy weighing $12 \mathrm{~kg}$ was admitted in infectious ward with trismus, difficulty in swallowing, intermittent muscle spastm, fever and purulent discharge from left ear from four days. He was not immunized for tetanus. He was diagnosed as otogenic tetanus. He received intramuscular tetanus toxoid, intravenous immunoglobulin 5000 IU as a bolus dose followed by 2500 IU/day and intravenous cefixime. Intravenous diazepam $6 \mathrm{mg}$ was given every three hours and intravenous midazolam was given $0.5 \mathrm{mg}$ if spasm was not relived. The ENT examination revealed the presence of foreign body and purulent discharge through left ear. On second day of admission, he was posted for removal of foreign body. He presented with intermittent muscle spasm, his pulse rate was $144 / \mathrm{min}$, blood pressure was $140 / 90 \mathrm{mmHg}$. He received intravenous diazepam $6 \mathrm{mg}$ and fentanyl $25 \mu \mathrm{g}$ as a premedication. He was preoxygenated for 5 minutes and induction was done with nitrous oxide, oxygen and sevoflurane. After complete relaxation, he was intubated with no. 5 plane endotracheal tube. Anesthesia was maintained on nitrous oxide, oxygen and sevoflurane. Left lateral position was given. $\mathrm{He}$ developed tachycardia and hypertension, with pulse rate $168 / \mathrm{min}$, blood pressure 160/94 mmHg. Deep plane of anesthesia was achieved with intravenous fentanyl $10 \mu \mathrm{gm}$. As tachycardia and hypertension was not controlled intravenous labetolol $3 \mathrm{mg}$ was given. Thereafter his blood pressure was $130 / 80 \mathrm{mmHg}$, pulse rate was $124 / \mathrm{min}$. The procedure was completed within 20 minutes. Two pieces of stone $3 \times 2 \mathrm{~mm}$ in size were removed. Extubation was uneventful. Patient was observed in post anesthesia care unit. He received intravenous diazepam $6 \mathrm{mg}$ every three hours and midazolam $0.5 \mathrm{mg}$ whenever required. After about couple of hours again he developed tachycardia and hypertension which was controlled by intravenous labetolol. His vitals became normal and no further betablocker therapy was required. The child was transferred to ward on next day.

\section{DISCUSSION}

Otogenic tetanus is a neurological disorder with spasticity, muscle spasms and autonomic disturbance caused by the neurotoxin-tetanospasmin secreted by Clostridium tetani from an otogenic source [4]. Incubation period of tetanus is three days to three weeks. It is classified into five grades according to presence or absence of five criteria, e.g., lock jaw, incubation period of seven days or less, presence of temperature of $100^{\circ} \mathrm{F}$ within 24 hours from development of lock jaw, presence of spasms and its period of onset within 48 hours or less. The mortality increases as the grade of severity increases [3]. The endotoxin affects nervous system. It has predilection for inhibitory neurons [2]. It binds to gangliosides and blocks the release of neurotransmitter from presynaptic inhibitory neurons. The loss of inhibitory impulses results in reflex irritability and autonomic hyperactivity. Centrally, transmission along the inhibitory gammaamino butyric acid (GABA) and glycinergic neurons is interrupted, and at the level of the spinal cord, inhibitory interneurons are blocked. Management includes administration of ant tetanus immunoglobulin, immunization, antibiotics and debridement of the wound, if needed. The objective of human immunoglobulin is to eliminate tetanospasmin that has not invaded the neurons so far $[1,5]$. Early treatment with immunoglobulin is critical, because it binds free toxin. However, it does not treat the effects of toxin protected within the neuron or cell body. Treatment of the effects of toxin already within the nervous system is purely supportive [5]. Initial management of muscle spasms involves sedation, isolation of patient in dark room, benzodiazepines, barbiturates, anticonvulsants, narcotics, baclofen, magnesium sulphate, dantrolene and propofol [2]. Benzodiazepines are often used as first-line treatment as GABA-A agonist thereby functioning as indirect antagonists of the effect of toxin on inhibitory system. Lorazepam has longer duration of action $[6,7]$. Midazolam is used as continuous infusion in patients of uncontrolled spasms [6]. Diazepam is preferred as it reduces muscle spasm by its effect on spinal interneuron and gamma motor neuron. It reduces central sympathetic activity [8]. Higher doses may be 
needed $(10-18 \mathrm{mg} / \mathrm{kg})$ by nasogastric tube in sever cases [8]. In case of failure to control spasm by benzodiazepines, nondepolarizing muscle relaxant is used. Nondepolarizing agents occupy the postsynaptic receptors, preventing acetylcholine neuromuscular transmission by competitive inhibiton and producing muscle relaxation. However, since there is heightened efferent neural discharge, generalized tetany markedly increases the requirement for nondepolarizing agents $[1,7]$. Neuromuscular blocking drugs with steroid molecule should be avoided in view of prolonged weakness [7]. Vecuronium infusion is free from cardiovascular side effects but may cause little histamine release [1].

Depolarizing neuromuscular blocker succinylcholine should be used with caution, as it may trigger hyperkalemic arrest. This may possibly be related acute renal failure leading to hyperkalemia or myoglobinuria. Dantrolene acts at the level of sarcoplasmic reticulum [6]. Baclofen is administered via the intrathecal route. It diffuses through capillaries of spinal cord and binds to GABA-B receptors in the substansia gelatinosa of dorsal horn to inhibit monosynaptic extensor and polysynaptic flexor transmission $[6,7]$. It carries a significant risk of respiratory depression [2]. Volatile anesthetic agents enhance the activity of inhibitory postsynaptic receptors while inhibit excitatory sympathetic channel activity [9]. Volatile anesthetics produce hypnosis, analgesia and inhibit motion, predominantly by acting at the level of the spinal cord. Sevoflurene relieves tetany and allows airway control and ventilation [9]. Supraglottic airway devices may further diminish the need for endotracheal intubation. As most air passes through the nasal passages during mask ventilation, a nasal airway can facilitate ventilation, even in the presence of masseter spasm. Magnesium with its unique property on the neuromuscular junction and sympathetic system has been used to treat both spasms and autonomic dysfunction with limited success $[6,7]$.

Magnesium reduces autonomic disturbances and spasm [10]. Magnesium blocks catecholamine release from nerves and adrenal medulla [7].

Autonomic dysfunction is the most serious complication of severe tetanus presenting with sustained but labile hypertension, tachycardia, arrhythmia, profuse sweating, pyrexia, increased carbon dioxide, increased catecholamine's and later on hypotension. These symptoms develop towards the end of first week [6]. Hypotension and bradycardia may also result from brainstem involvement or myocarditis [9]. Autonomic hyperactivity is treated with narcotics which also relieves pain [11]. Labetalol acts by inhibiting uptake of norepinephrine into nerve terminals. It can be helpful along with sedatives and narcotics. We used intravenous fentanyl, midazolam and bolus dose of labetolol but we did not require continuous infusion of labetolol. Epidural blockade is effective in controlling sympathetic over activity and the associated complications [12]. Beta-blockers should be used with caution as they have been implicated in the deaths of some patients with autonomic dysfunction [7]. In treating Clostridium tetani infection, metronidazole is more effective than Penicillin G since it is a GABA antagonist [12]. Even though there is no documented evidence regarding safety of volatile anesthetic agents we used sevoflurane as a sole agent as the procedure is of short duration and in our patient muscle spasm was very well controlled.

\section{CONCLUSION}

In case of tetanus, volatile anesthetic agents can be safely used for surgical procedures like cleaning and debridement of wound or incision and drainage of abscess. However, use of nondepolarizing muscle relaxants is advisable. As we used sevoflurene in single case, further study is required for confirmation of its efficacy and safety. Autonomic hyperactivity can be managed with beta-blocker, intravenous labetolol is the drug of choice.

$* * * * * * * * *$

\section{Author Contributions}

Jyoti V Kulkarni - Conception and design, Acquisition of data, Analysis and interpretation of data, Drafting the article, Critical revision of the article, Final approval of the version to be published

Anil Shriniwas Joshi - Conception and design, Acquisition of data, Analysis and interpretation of data, Drafting the article, Final approval of the version to be published

Rashmi V Bengali - Conception and design, Acquisition of data, Analysis and interpretation of data, Drafting the article

Suhas Jewalikar - Conception and design, Acquisition of data, Analysis and interpretation of data, Drafting the article

\section{Guarantor}

The corresponding author is the guarantor of submission.

\section{Conflict of Interest}

Authors declare no conflict of interest.

\section{Copyright}

(C) Jyoti V Kulkarni et al. 2013; This article is distributed under the terms of Creative Commons Attribution 3.0 License which permits unrestricted use, distribution and reproduction in any means provided the original authors and original publisher are properly credited. (Please see www.ijcasereportsandimages.com/copyright-policy.php for more information.)

\section{REFERENCES}

1. Paul Firth, John Bastien, Boston, Table \#36 PBLD: Tetanus: Anesthetic management during a mass casualty natural disaster SPA 2011. 
2. Pramila Bajaj. Tetanus: Anaesthetic Management. Indian journal of Anaesthesia 2009;53(3):367-8.

3. Patel JC, Mehta BC. Tetanus: study of 8,697 cases. Indian journal of medical sciences 1999;53(9):393-401.

4. Akinbohun A, Ijaduola G. Otogenic Tetanus among Children in Ibadan, Nigeria. The Internet Journal of Otorhinolaryngology 2009:10(2).

5. Gibson K, Bonaventure Uwineza J, Kiviri W, Parlow J. Tetanus in developing countries: a case series and review. Can J Anaesth 2009;56(4):307-15.

6. Talaat Abdelmoneim, Lucian K DeNicola, M Yousuf Hasan. Tetanus: Complications and Management in a Paediatric Intensive Care UnitThe Division of Paediatric Critical Care, University of FloridaJacksonville.

7. Reddy VG. Pharmacotherapy of tetanus-a review. Middle East J Anaesthesia 2002 Feb;16(4):419-42.

8. F. D. Dastur, chapter- II -15 Clostridial infection, API Textbook of Medicine seventh edition (Editor in chief Siddharth N Shah). 39-43.
9. Firth PG, Solomon JB, Roberts LL, Gleeson TD. Airway management of tetanus after the Haitian earthquake: new aspects of old observations. Anesth Analg 2011;113(3):545-7.

10. Alok Kumar, Raktima Anand, Anita Rahal, Sandhya Od. Use of intrathecal and intravenous clonidine in case of severe tetanus with acute renal failure. Indian J Anaesth 2009 Apr;53(2):204-8.

11. Powles $\mathrm{AB}$, Ganta $\mathrm{R}$. Use of vecuronium in the management of tetanus. Anaesthesia 1985;40(9):879-81.

12. Bhagwanjee S, Bösenberg AT, Muckart DJ. Management of sympathetic over activity in tetanus with epidural bupivacaine and sufentanil: experience with 11 patients. Critical care Medicine 1999 Sept;27(9):1721-5.
Access full text article on other devices

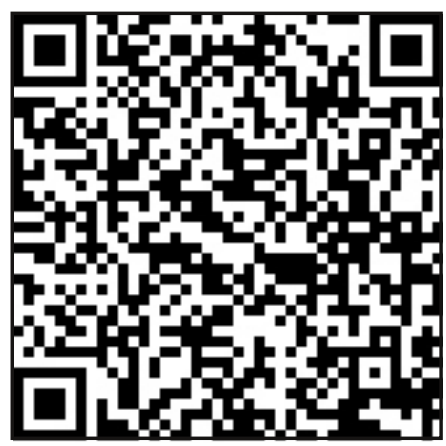

Access PDF of article on other devices

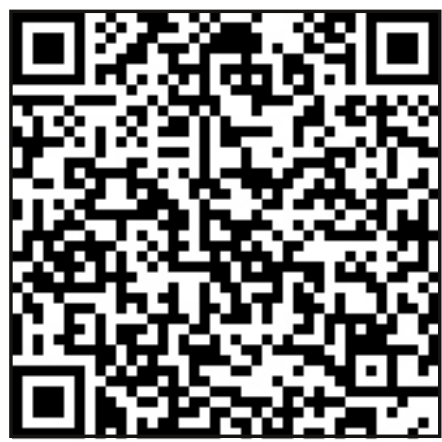

